\title{
UMANESIMO REPUBBLICANO, UMANESIMO CORTIGIANO. L'ITALIA DEL TRE-QUATTROCENTO
}

\author{
Marcello Fantoni \\ (Kent State University) \\ mfantoni@kent.edu
}

\section{RIASSUNTO}

L'umanesimo cortigiano costituisce il fondamento di un processo che, portando alla formulazione di una nuova cultura in Italia, conferirà a questa le caratteristiche che ne determineranno l'irradiamento europeo sulla base di una forza catalizzatrice che è eminentemente politica. Le 'piccole' corti italiane del Quattrocento funzionano, in questo, da incubatrici dei nuovi modelli di civilizzazione per le grandi corti europee dell'Antico regime.

PAROLE CHIAVE: Umanesimo, corte, Italia, Quattrocento, Antico regime.

\section{REPUBLICAN HUMANISM, COURT HUMANISM. THE ITALY OF THE TRE-QUATTROCENTO}

\begin{abstract}
Court humanism constitutes the foundation of a process that, leading to the formulation of a new culture in Italy, will give it the characteristics that will determine its European radiation based on a catalytic force that is eminently political. In this context, the 'small' Italian courts of the Quattrocento function as incubators of new civilization models for the great European courts of the Old Regime.
\end{abstract}

KEY WORDS: Humanism, court, Italy, Quattrocento, Old Regime.

Secondo una nobile tradizione che discende principalmente dallo storico berlinese Hans Baron (ma le cui origini risalgono ancora più addietro), umanesimo e repubblicanesimo costituiscono due termini invariabili di una stessa equazione. Dagli anni Cinquanta del secolo scorso, questa è stata l'interpretazione di almeno tre generazioni di storici (soprattutto italiani e anglosassoni) che si sono avvicendate negli archivi di Venezia e di Firenze alla ricerca delle radici della propria civiltà e di autorevoli antenati della modernità. Per quasi settanta anni, nel bene o nel male, il libro di Baron ha dato vita a un coro di risposte, critiche, rettifiche e ampliamenti che hanno 
comunque sortito l'effetto di tenere impegnati gli storici sull'argomento dell'umanesimo civile ${ }^{1}$. È questa progenie di studi che ha fatto di Baron la figura di riferimento di una certa interpretazione politica del Rinascimento italiano. Visti i tempi in cui maturarono le convinzioni e in cui scriveva Baron - fra la crisi della Repubblica di Weimar e l'ascesa del nazismo, la Seconda Guerra Mondiale e l'inizio della Guerra Fredda - si comprende il perché della sua visione. I problemi sono altri. In primis il fatto che la storiografia ha stazionato a lungo su questi temi senza che sia mai avvenuta una loro storicizzazione e secondo che, per questo, ancora oggi esistono epigoni di questa visione, il che, alla luce delle attuali circostanze, risulta meno comprensibile.

Vediamone alcune di queste "reazioni" alla pubblicazione di The Crisis of the Early Italian Renaissance (1955). Fra riserve metodologiche e distinguo ideologici, non abbiamo che l'imbarazzo della scelta, fra recensioni e vere e proprie monografie, ampliamenti di prospettiva, e estremismi che arrivano a negare en bloc l'esistenza di un umanesimo civile ${ }^{2}$. Lo stesso Paul Oskar Kristeller, che nelle linee generali accetta le tesi baroniane, rammenta tuttavia che sarebbe un errore identificare tutto l'umanesimo con l'umanesimo civico fiorentino e - seppur con accento negativo - ricorda l'esistenza di un «despotic humanism» ${ }^{3}$. Quello fiorentino è solo un tipo fra i molti di umanesimo sia in senso geografico, che politico o tematico. Un rimprovero mosso da più parti mette inoltre in dubbio l'affermazione che Firenze sia tout court la "cradle of humanism", questo ad avvalorare invece un quadro più articolato e diffuso del pensiero umanistico fra le molte realtà urbane e politiche della penisola. A corollario di questo primo argomento, un'altra obiezione ricorrente contesta il fatto che esista un ipotetico circolo virtuoso fra umanesimo, repubblicanesimo e fiorire delle arti e della cultura.

Negli anni 1400-1402 si è inoltre indicata una congiuntura estrema che avrebbe spinto a redigere opere propagandistiche in difesa della repubblica, ma proprio per questo si tratterebbe di posizioni dettate dalla specificità delle circostanze e - dunque non si potrebbe veramente parlare di ordinario pensiero umanistico. In altre parole, sono gli umanisti stessi, nella drammaticità del momento, a creare l'idea di Firenze come città della libertà, unica rimasta a difendere lo stendardo di fronte all'espansionismo di Gian Galeazzo Visconti. Questi sono, come sappiamo, gli intenti della Laudatio Florentinae Urbis e del Dialogo a Pier Paolo Vergerio di Leonardo Bruni, e questa le finalità della Istoria di Firenze, 1380-1406 nella quale Gregorio Dati afferma che le libertà dell'Italia tutta sono nelle mani di Firenze. Ė invece proprio dando credito a queste affermazioni che Baron giunge a ipotizzare il contributo essenziale offerto dall'umanesimo civile allo sviluppo politico-culturale dell'occidente. Senza contare il fatto, e anche su questo si sono appuntate le critiche, che lo stesso governo di Firenze

\footnotetext{
${ }^{1}$ Cfr. Hans Baron, The Crisis of the Early Italian Renaissance. Civic Humanism and Republican Liberty in an Age of Classicism and Tyranmy (Princeton: Princeton University Press, 1955). Per una sintetica rassegna delle diverse posizioni riguardo a Baron vedi Albert Rabil, Jr., ed., Renaissance Humanism. Foundations, Forms and Legacy. I. Humanism in Italy (Philadelphia: University of Pennsylvania Press, 1988), 141-179.

2 Su questo vedi Jerrold Seigel, “'Civic Humanism' or Ciceronian Rhetoric? The Culture of Petrarch and Bruni”, Past and Present 34 (1966): 3-48, https://doi.org/10.1093/past/34.1.3.

${ }^{3}$ Cfr. Paul Oskar Kristeller, Renaissance Thought II. Papers on Humanism and the Arts (New York: Harper Torchbooks, 1965), 46-47.
} 
non rappresentava affatto una incarnazione di questo spirito di libertà, e non solo per la diversa semantica attribuita al termine libertas, ma per l'effettiva natura oligarchica del regime, ancor prima dell'ascesa della famiglia Medici.

Inizia anche ben presto la campagna di verifiche storiche del background sociale, economico e politico delle tesi di Baron, il che da un lato mette in evidenza alcuni dei suoi limiti, ma che dall'altro comunque contribuisce a incentrare la scholarship sull'esempio fiorentino come laboratorio in cui studiare in vitro le casistiche che si ritrovano nella contemporaneità. Fra i primi a scendere in campo è Marvin Becker, il quale in un'opera pionieristica del 1967-1968 attribuisce la genesi dell'umanesimo civico - piuttosto che alle impellenze della guerra contro i Visconti - alle sollecitazioni della sfera fiscale. Il deficit del debito pubblico a partire dagli anni Venti del Trecento, che conduce alla istituzione del Monte nel 1345 e alle successive ristrettezze finanziarie della città, porta i ceti dirigenti - questa la spiegazione di Becker - a fare quadrato intorno al regime cittadino. In questo clima, si inscrive il richiamo di Bruni alla vita civile, nell'ambito cioè della condivisione delle sorti fra ceto mercantile e sopravvivenza dello stato fiorentino ${ }^{4}$. Qualche anno prima Lauro Martines aveva a sua volta enfatizzato una prospettiva per la quale l'umanesimo sarebbe invece il frutto e l'espressione dell'élite socio-politica, con gli umanisti stessi che provengono in prevalenza da questo gruppo, che questo gruppo rappresentano in seno alle istituzioni e che ad esso e al governo che lo incarna danno validazione nei loro scritti ${ }^{5}$. Gli umanisti appartengono in altre parole alla "ruling class" fiorentina e i vari Marsuppini, Bruni, Alberti, Rinuccini, Salviati, ecc., ne sono la voce soprattutto dopo le strette oligachiche successive alla rivolta dei Ciompi (1378). Queste circostanze si ripercuotono sulla rappresentazione del regime politico che assume i connotati di interesse di classe. Le virtù di cui trattano gli umanisti sono dunque le stesse che supportano il potere e che sostengono la loro posizione di privilegio al suo interno. Sempre secondo questa logica, non sorprende dunque che con la fine di questo sistema e con l'avvento dei Medici nel 1434, all'umanesimo civile si sostituisca il docilmente schierato neoplatonismo.

Con questo arriviamo agli anni Settanta, forse l'apogeo di quella che nelle università nord-americane prenderà il nome di "fiorentinistica". Fra gli innumerevoli studi che fanno la loro comparsa in questo decennio, quello di Gene Brucker su The Civic World of Early Renaissance Florence (1977) è senz'altro il più degno di nota e il più strettamente legato all'opera baroniana. Anche in questo caso si tratta di una parziale rettifica, in particolare in senso politico. Brucker vi sostiene infatti che fra 1378 e 1434 (fra i Ciompi e i Medici) - quasi esattamente un cinquantennio - bisogna guardare alle forme istituzionali, completando con questo quelle che a suo avviso sono le letture

\footnotetext{
${ }^{4}$ Cfr. Marvin Becker, Florence in Transition, 2 voll. (Baltimore: Johns Hopkins University Press, 19671968), https://doi.org/10.1353/book.67860, https://doi.org/10.1353/book.67859. Questa tesi viene anche sostenuta in Marvin Becker, "The Florentine Territorial State and Civic Humanism in the Early Renaissance", in Florentine Studies. Politics and Society in Renaissance Florence, ed. Nicolai Rubinstein (Evanston: Northwestern University Press, 1968), 109-139.

${ }^{5}$ Vedi Lauro Martines, The Social World of the Florentine Humanists, 1390-1460 (Princeton: Princeton University Press, 1963).
} 
parziali di Becker e di Martines. Per questa via si arriva ancora al civic humanism, questa volta espressione della transizione dal «corporate» all'«elitist state» ${ }^{6}$. In tutti i casi, comunque, si ridimensiona l'impatto del moto patriottico che si sarebbe collegato al critico biennio di guerra 1400-1402.

Due importanti conferme e dilatazioni di contesto vengono, sempre negli anni Settanta, da Quentin Skinner e da J. G. A. Pocock (due storici del pensiero politico). Il primo per il suo ricostruire gli antefatti due-trecenteschi dell'umanesimo civile e per il suo inserimento in una prospettiva di lunga maturazione. In particolare Skinner sostiene che «what Baron believed to be new in the early Quattrocento was in fact inherited» dalla tradizione preumanistica e scolastica delle «city republics of medieval Italy» ${ }^{7}$. Il secondo, sul versante cronologico opposto, per sostenere il riaffiorare ciclico di questi umori filosofici nella Glorious Revolution e nella Rivoluzione Americana, e dunque nel cementare il percorso della moderna democrazia in un presupposto "asse atlantico" del pensiero repubblicano. Skinner spingendosi indietro nel tempo e Pocock in avanti - guardando alle parentesi repubblicane del 1494-1512 e del 1527-1530 - in definitiva legittimano l'umanesimo civile entro il generale processo politico dell'occidente $^{8}$. Il mito è servito! E quello che sorprende del paradigma repubblicanoumanistico è (come abbiamo già accennato) il suo perdurare sebbene il contesto storico - dopo la caduta del muro di Berlino e l'affermarsi di un fluido multipolarismo - sia ormai profondamente mutato, e sebbene il succedersi degli studi abbia evidenziato più di un elemento di debolezza.

L'Italia tardo-medievale e primo-rinascimentale costituirebbe dunque il luogo di nascita di quel repubblicanesimo municipale da cui derivano genealogicamente le democrazie occidentali. Tesi che si è (o dovrebbe essersi) tramutata in mito poiché la sua veridicità è stata da tempo «de-masked» ${ }^{9}$. Si parte dagli studi di Cecilia Ady sulla Bologna dei Bentivoglio (siamo nel 1937), attraverso quelli di Nicolai Rubinstein sul significato di libertas (di qualche decennio successivi), fino alle più recenti pagine di Alison Brown, e alle ormai numerose opere sulle signorie tre-quattrocentesche, con in testa quelle di Philip Jones ${ }^{10}$. Non ci dovrebbe dunque essere bisogno di provare ulteriormente l'inconsistenza di questo paradigma. C'è abbondanza di letteratura sull'argomento, da come il mito viene fabbricato dagli stessi umanisti fiorentini, a come di esso si appropri la recente storiografia. A un esame più ravvicinato risulta del resto anche che le città-stato comunali e i regimi principeschi non sono politicamente e culturalmente antitetici. I confini tra di loro sono assai più sfumati di quanto non ci

\footnotetext{
${ }^{6}$ Vedi Gene Brucker, The Civic World of Early Renaissance Florence (Princeton: Princeton University Press, 1977), https://doi.org/10.1515/9781400847853.

${ }^{7}$ Rabil, ed., Renaissance Humanism, 157.

${ }^{8}$ Le opere in questione sono in particolare Quentin Skinner, The Foundations of Modern Political Thought. I. The Renaissance (Cambridge: Cambridge University Press, 1978), https://doi.org/10.1017/CBO9780

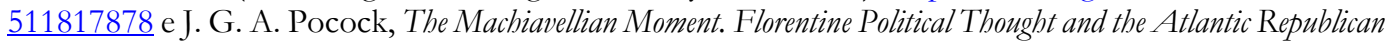
Tradition (Princeton: Princeton University Press, 1975).

${ }^{9}$ The expression is borrowed from Allison Brown, "De-masking Renaissance Republicanism”, in Renaissance Civic Humanism. Reappraisals and Reflections, ed. James Hankins (Cambridge: Cambridge University Press, 2000), 179-199, https://doi.org/10.1017/CBO9780511558474.007.

${ }^{10}$ Cfr. Philip Jones, The Italian City-State. From Commune to Signoria (Oxford: Clarendon Press, 1997).
} 
abbia voluto far credere la coeva propaganda politica. Proprio gli umanisti ne sono - ironicamente - una prova inconfutabile. Allora perché insistere sul tema? Non c'è il rischio di sfondare porte aperte? Penso di no, o - almeno a mio parere - non del tutto. Primo perché sopravvive una frangia di 'repubblicanisti' - neo-progressisti - che si ostinano a sostenere questa lettura tautologica. Secondo, perché manca ancora una pars costruens, vale a dire una ricerca che delinei gli elementi di autenticità del nuovo scenario.

Veniamo al dunque. L'Italia del periodo in questione presenta un quadro politico poliedrico - non una polarizzazione in bianco e nero fra signorie e comuni. $\mathrm{Al}$ suo interno si ha inoltre una netta prevalenza delle prime e dunque di sistemi incentrati sull'istituzione della corte (più o meno sviluppata essa sia). Soprattutto, ed è questo che ci interessa, il quadro nel suo complesso è intriso di umanesimo. L'umanesimo non è cioè una prerogativa intellettuale dei regimi repubblicani. Inoltre, non solo le corti ne sono centri di eguale rilievo, ma esse fungono anche da incubatrici della sua maturazione in classicismo, vale a dire nel modello culturale che d'avvero costituisce il carattere fondativo della modernità. Il mondo antico è per tutti un'inesauribile e autorevole serbatoio di exempla, testi, valori, eroi. Tutti prendono a prestito dall'antichità in cerca di argomenti convalidanti il proprio presente e tutti setacciano gli stessi testi. Il De Officis di Cicerone ispira sia Brunetto Latini quando dichiara il governo repubblicano la migliore costituzione politica sia Baldassarre Castiglione nel delineare i tratti del perfetto cortegiano. L'Etica e la Politica di Aristotele sono la fonte per gli Specula Principis, della dottrina repubbicana e dei sostenitori del sistema imperiale. Quintiliano è il referente per la retorica di ogni parte, Plauto per il teatro popolare e colto, Vitruvio per tutta l'architettura. E potremmo continuare.

L'antichità è indistintamente l'interlocutrice di tutti i regimi politici; gli ideali cesariani e repubblicani sono riesumati alternativamente dall'antichità romana a seconda delle necessità e situazioni. C'è solo una rinascita degli antichi ubiquamente ispirante il pensiero politico del XIV e XV secolo. Ambedue i sistemi fondano la propria legittimazione sull'autorità dei classici (gli umanisti sono uniti dall'amore per il mondo classico); si tratta semplicemente di trovare, e rifunzionalizzare, quello che meglio serve alle loro rispettive ideologie. Sebbene, ovviamente, fra Trecento e Quattrocento tante sono le ramificazioni, le correnti e le variabili geografiche e temporali, l'umanesimo cerca comunque di elevare la qualità morale e intellettuale del potere. Sia le repubbliche che le signorie utilizzano un simile repertorio retorico, riconoscono l'insuperata autorità dell'eredità greca e romana, sono profondamente immerse in essa, e ugualmente promuovono il riuso delle categorie del passato per costruire le fondamenta del loro futuro.

In aggiunta gli umanisti italiani sono una omogenea élite intellettuale unita da comuni interessi di studio, una fitta rete epistolare, un incessante viaggiare e destini personali frequentemente intrecciati. Si spostano di continuo e, sia con legami di amicizia che con diatribe polemiche, sono strettamente legati gli uni agli altri. Scrivono tutti commentari, orazioni funebri, relazioni diplomatiche, storie e biografie, e condividono gli stessi orizzonti intellettuali, piegando - semplicemente - la semantica dei temi classici a seconda delle loro diverse inclinazioni o ambienti di appartenenza. 
Concetti come virtus oltrepassano i confini politici e sono associati ai codici morali che guidano qualsiasi azione umana o sociale. Lo stesso termine libertas non è antinomico rispetto ai regimi monarchici, riferendosi all'autonomia politica della compagine statuale. Allo stesso modo la denominazione di princeps è indifferentemente usata per connotare il governo di un comune o di un principe. Il revival della nozione aristotelica di magnificentia operato da Pontano, sebbene sviluppato alla corte di Alfonso il Magnanimo a Napoli, sta indistintamente alla base del patronage di generazioni di mercanti, aristocratici e signori ${ }^{11}$. Infine, la prudentia, si trova intessuta sia nelle teorizzazioni monarchiche che repubblicane, è essenziale per chiunque occupi uffici pubblici e costituisce materia prima nel delineare i contorni del buon governo.

L'umanesimo è la filosofia, l'ecosistema culturale, e il comune universo mentale di un'intera epoca. Gli stessi umanisti provengono da un comune background, sono egualmente immersi nella vita civile e negli studia (si sforzano di unire vir sapiens e virfaber in una vita attiva volta al conseguimento del bonum comune) e - inoltre - sono cristiani e coniugano fede e classici nelle loro esperienze personali e nei loro impegni civili. Il riuso dei classici non ha valenza anti-religiosa. Come già ammoniva Konrad Burdach nel 1935: «L'Umanesimo non è mai esistito come un tutto organico concluso» e, soprattutto, questo movimento non ha niente di pagano «e assolutamente nulla in comune» - come vorrebbe invece Baron - «con la libertà politica intesa in senso moderno, nel senso del liberalismo europeo derivato dalla Rivoluzione francese» ${ }^{12}$. "Forma del vivere" aristocratica ed etica borghese, laicismo e cristianesimo sono pertanto compresenti ed amalgamati: il che dà vita a un Rinascimento più ricco e sicuramente diverso da quanto si è spesso dipinto.

Gli esempi sono (e sono stati) del resto sotto gli occhi di tutti, sia quelli relativi ai singoli umanisti che alle loro opere. Vediamone - sommariamente - alcuni, quanto basta per tratteggiare il quadro di riferimento. Intanto il latino viene ritenuto la lingua più adatta a esprimere il pensiero degli umanisti; e la retorica e l'eloquenza classica sono imitate per conferire efficacia argomentativa ai loro scritti o pregnanza ai loro discorsi. L'umanista di origini ferraresi Tito Livio Frulovisi (1400-1480) osserva nel suo De repubblica che se «l'eloquenza è necessaria ai cittadini con responsabilità di governo», a maggior ragione essa è «importante per il principe signore di una città» ${ }^{13}$. Essa serve in ogni caso a qualsiasi ceto dirigente di qualsiasi sistema costituzionale. Quella stessa eloquenza - tratta dalle stesse fonti - che, poi, nel Cortegiano (1528) si trasformerà in conversazione e diventerà un'arte del gentiluomo. Questa è la ragione per cui i titolari di scuole umanistiche la includono nei propri curricula e danno importanza alla gestualità e alla grazia dell'oratoria.

Sempre e comunque li unisce la politica. Tanti umanisti sono signori e sovrani essi stessi, come Alberto III Pio da Carpi, Pico della Mirandola, Federico da

${ }^{11}$ Cfr. Giovanni Pontano, De Magnanimitate [1498], ed. Francesco Tateo (Firenze: Istituto Nazionale di Studi sul Rinascimento, 1969).

12 Konrad Burdach, Riforma, Rinascimento, Umanesimo [1935] (Firenze: Sansoni, 1986), 75, 120. Sul tema si è espresso anche Carl Trinkaus, In Our Image and Likeness. Humanity and Divinity in Italian Humanist Thought, 2 voll. (Chicago: University of Chicago Press, 1970).

${ }^{13}$ Lauro Martines, Potere e fantasia. Le città stato nel Rinascimento [1979] (Roma-Bari: Laterza, 1981), 254. 
Montefeltro, Leonello d'Este, Pandolfo Malatesta, Lorenzo de' Medici, e molti altri. E con Pio II Piccolomini (1458-1464) ancora una volta, dopo il pontificato di Niccolò V Parentucelli e prima di quello di Leone X de' Medici, la cultura umanistica sale sulla cattedra di Pietro. Come umanisti si comportano nel mecenatismo, nella creazione di biblioteche e nel circondarsi di sodali, e come umanisti sono anche rappresentati nella ritrattistica di stato che mira a identificarli con le lettere e non più esclusivamente con le armi.

Il movimento umanistico è ovunque a corte con le sue scuole e con il circolare dei suoi testi, uomini e saperi. Entro questa sostanziale omogeneità, non pochi sono tuttavia i suoi caratteri distintivi, per il suo adattarsi ai diversi ambienti socio-politici. Riguardo all'umanesimo napoletano, si è in tal senso messa in evidenza la concomitanza fra cosmopolitismo e problematiche proprie del regno aragonese ${ }^{14}$. Per ricoprire le mansioni diplomatiche, Alfonso il Magnanimo chiama a Napoli uomini di riconosciuta fama come il Panormita, Bartolomeo Facio, Lorenzo Valla, Manetti e Giovanni Pontano. Costoro, e molti altri, consacreranno le proprie energie al servizio della corona, attingendo alla propria perizia filologica e conoscenza dei filosofi e degli storici antichi. Dal secondo Quattrocento, con il ritorno della sede apostolica nell'urbe, Roma diventa a sua volta una delle destinazioni più prestigiose per gli umanisti italiani; al servizio della curia pontificia troviamo così - anche con effetto drenante da Firenze - Coluccio Salutati, Giannozzo Manetti, Gasparino Barzizza, Antonio Loschi, Leonardo Bruni, Pier Paolo Vergerio, Leon Battista Alberti, Poggio Bracciolini, Giovanni Aurispa, solo per citare i nomi più illustri.

Gli umanisti occupano comunque posizioni di rilievo nelle cancellerie, servono come diplomatici, sono segretari e cortigiani. Spesso ricoprono più di uno di questi ruoli nel corso della loro vita o detengono molteplici cariche allo stesso tempo. In questo mutevole scenario, non era insolito per gli umanisti di cambiare 'parte', appartenendo alternativamente a magistrature comunali e servendo nelle corti, o amministrazioni di principi, cardinali e papi. Gli umanisti sono liberi professionisti sul mercato della politica; sono intellettuali al potere, potere che essi istruiscono e amministrano. Evidente è la capacità degli umanisti di entrare in simbiosi con il contesto politico in cui si trovano ad operare. Tutti, quale che sia il loro schieramento, sono alleati del potere, servono e partecipano al potere, scrivono indistintamente panegirici di signori e lodi di città. Soprattutto essi sono ben consapevoli di possedere una merce richiesta dal mercato. Ogni umanista ha le sue tariffe e ogni città e signore ha i suoi tetti di spesa. L'umanesimo è la maggiore esperienza intellettuale delle classi dirigenti.

Alcuni di essi sostengono un regime per la loro intera esistenza, ma molti di più sono coloro che non si peritano dall'offrire il loro sapere, e le loro abilità diplomatiche, oratorie e letterarie al miglior offerente. Il fiorentinissimo Alberti è un assiduo frequentatore delle corti: è a Roma nel 1432, nel 1434 è nel seguito di papa Eugenio IV, nel 1436 lavora per Borso d'Este a Ferrara, dal 1444 è di nuovo a Roma e

${ }^{14}$ Cfr. Jerry Bentley, Politics and Culture in Renaissance Naples (Princeton: Princeton University Press, 2014), 295 e sgg., https://doi.org/10.1515/9781400858811. 
collabora con Nicolò V, nel 1450 progetta il tempio malatestiano di Rimini, tra 1463 e 1471 disegna le chiese mantovane di San Sebastiano e Sant'Andrea, e muore a Roma nel 1472. Pier Paolo Vergerio si sposta continuamente fra la Bologna dei Bentivoglio, la Firenze repubblicana (dove studia greco con Manuele Crisolora), la Padova dei da Carrara, e Roma dove fu segretario dei papi Innocenzo VII (1404-1406) e Gregorio XII (14061409). Giannozzo Manetti, mercante fiorentino (suo padre Bernardo era uno dei dieci uomini più ricchi di Firenze), dopo aver lasciato gli affari, ha una carriera che lo porta a servire presso la curia pontificia e la corte napoletana. Nel corso del 1453 Manetti lascia il territorio della repubblica in due occasioni. La prima a inizio anno, in fuga dalle vessazioni fiscali lui imposte da Luca Pitti e la seconda alla fine dell'anno come «congedo definitivo da Firenze» ${ }^{15}$. Dopo aver servito fedelmente in innumerevoli cariche pubbliche, Manetti si mette dunque al servizio di papa Niccolò V e di re Alfonso d'Aragona, il primo dei quali celebra nella nota De vita ac gestis Nicolai Quinti summi pontificis, e il secondo al quale dedica il suo De dignitate et excellentia hominis. Atto quest'ultimo, che - compiuto nel pieno della guerra fra Firenze e Napoli - gli procurerà una condanna al confino, poi revocata per il provvidenziale intervento del pontefice che ne richiede al contempo il trasferimento a Roma. La Signoria non aveva poi tutti i torti, poiché nel De dignitate Manetti vede in Alfonso, acerrimo nemico fiorentino, «lincarnazione vivente del sovrano ideale» ${ }^{16}$. Ancora ad Alfonso (dal quale riceve un lauto salario di novecento ducati annui) dedicherà l'adesso perduto Vita Alfonsi regis. A Roma, a fine del 1458, Manetti ricoprirà infine la carica di segretario apostolico da papa Pio II. In questo ondivagare - più che paladino della repubblica fiorentina - sembra dunque appropriato dire di Manetti che la sua «ideology can be summed up as follows: power should be celebrated, regardless of its form, as long as law and order are preserved in defense of the Christian faith and in the interest of the mercantile class» ${ }^{17}$.

Il mutare degli schieramenti con la transizione fra Visconti e Sforza e con la nuova alleanza antiveneziana fra Milano e Firenze, trasforma Filelfo da «baldo difensore della 'florentina libertas' in un letterato cortigiano» ${ }^{18}$. Ferretto Ferretti $(† 1337)$ inizia a scrivere come un sostenitore della repubblica e finisce a redigere elogi dei della Scala - signori di Verona - nel suo poema De Scaligerorum origine, dove sottolinea il loro ruolo di pacificatori della civitas $^{19}$. Nel suo poema incompiuto Africa, Petrarca dipinge l'Imperium di Cesare «almost exclusively in its darkest aspects», ma successivamente nel De Gestis Cesaris non si trova più «a trace of his former censure of

\footnotetext{
${ }^{15}$ Luca Boschetto, "L'esilio volontario di Manetti", in Dignitas et excellentia hominis. Atti del Convegno Internazionale di Studi su Giannozzo Manetti, ed. Stefano Baldassarri (Firenze: Le Lettere, 2008), 117-146, 118. Vedi anche Mario Martelli, "Profilo ideologico di Giannozzo Manetti”, Studi Italiani 1 (1989): 5-41.

${ }^{16}$ Fabrizio Ricciardelli, "Giannozzo Manetti, un intellettuale al potere", in Dignitas et excellentia hominis. Atti del Convegno Internazionale di Studi su Giannozzo Manetti, ed. Stefano Baldassarri (Firenze: Le Lettere, 2008), 279-300, 289.

17 Stefano Baldassarri, "Introduction", in Giannozzo Manetti. Biographical Writings, ed. Stefano Baldassarri e Rolf Bagemihl (Cambridge-Londra: Harvard University Press, 2003), VII-XIX, XIII.

${ }^{18}$ Eugenio Garin, "La letteratura degli umanisti", in Storia della Letteratura Italiana. III. II Quattrocento e l'A riosto (Milano: Garzanti, 1966), 7-353, 118.

${ }^{19}$ Jones, The Italian, 644.
} 
the Empire» ${ }^{20}$ (lo dice lo stesso Baron). Molti umanisti cambiano disinvoltamente città, protettori e parte politica. L'aretino Leonardo Bruni (1370-1444) fu cancelliere dei Signori a Firenze e secretaries apostolicus a Roma, e non trova improprio dedicare una sua opera ad Alfonso il Magnanimo. Il suo maestro, Coluccio Salutati, serve lealmente come cancelliere della repubblica Fiorentina fra il 1376 e la sua morte nel 1406. Ciò nonostante, proprio nel bel mezzo il drammatico conflitto con i Visconti, completa il suo De Tyranno (1400) nel quale delinea i caratteri del despota benevolo. Sebbene il tiranno «sia la peggior piaga che possa infettare il corpo politico» un sovrano benigno è il solo rimedio all'impotenza del comune per garantire la giustizia; e dunque - conclude Salutati - «la monarchia è da preferire a ogni altra forma di governo [...]. Non esiste maggior libertà dell'obbedienza al giusto comando di un principe virtuoso» ${ }^{21}$. Un secolo prima del Principe di Machiavelli, Salutati definisce inoltre redimendo Cesare - la differenza fra il tiranno e il signore legittimo. Il

tiranno è sia colui che usurpa il potere, senza titolo legittimo per la sua autorità, o colui che governa superbe o ingiustamente o non osserva la legge e l'equità: proprio come, dall'altro lato, un principe legittimo è colui al quale il potere viene conferito, che amministra la giustizia e mantiene la legge ${ }^{22}$.

Come abbiamo visto, una guerra propagandistica di pamphlets si scatena negli anni critici del conflitto Firenze-Visconti. Le ostilità si aprono con l'Invectiva in Florentinos (1397) di Antonio Loschi e - come sappiamo - la sfida viene raccolta da Leonardo Bruni, oltre che da Salutati. In un contesto di netta contrapposizione nel 1436, dopo aver composto una vita di Filippo Maria Visconti, Pier Candido Decembrio sente la necessità di scrivere un De laudibus Mediolaniensium Urbis in comparationem Florentiae panegyricus. Eppure, nonostante le loro ferme posizioni, Loschi resta allievo di Salutati e amico di Bruni. Proprio Salutati, anzi, lo raccomanda per la carriera cancelleresca alla corte milanese. E anche Uberto Decembrio da Vigevano dopo il 1407 mette la sua penna al servizio della pax italica viscontea, pur continuando a venerare apertamente la gloria di Salutati.

Dopo l'esperienza fiorentina come notaio, Giovanni Conversini da Ravenna risiede alla corte di Francesco il Vecchio da Carrara del quale si guadagna stima e simpatia. Apre una scuola a Venezia nel 1388, ma accetta nuovamente un invito dei da Carrara per insegnare all'università di Padova nel 1392. Da qui la sua fama arriva ben presto a corte e nel 1393 Francesco Novello lo vuole al suo fianco come cancelliere. Nel 1396 scrive il De fortuna aulica, lodando la vita di corte, e tre anni dopo l'Apologia, questa volta lamentando le invidie dei cortigiani ${ }^{23}$. Sulla base della sua quarantennale

20 Baron, The Crisis, 56, 57.

${ }^{21}$ Coluccio Salutati, De Tyranno, in Ephraim Emerton, Humanism and Tyranny. Studies in the Italian Trecento (Cambridge: Harvard University Press, 1925), 92, 108, https://doi.org/10.4159/harvard.97806 74333178 .

${ }^{22}$ Vedi ibidem, 78.

${ }^{23}$ Cfr. Giovanni Conversini da Ravenna, Two Court Treatises, ed. Benjamin G. Kohl e James Day (Monaco: Wilhelm Fink Verlag, 1987), 15-20, 84-91. 
esperienza, alla corte Conversini dedicherà altri due trattati: il De Primo eius introitu ad aulam (1385) e il De dilectione Regnantium (1399). La sua non sempre fortunata vita peripatetica lo porta anche alla corte di Niccolò II d'Este come istitutore (1366) e tre anni dopo all'insegnamento presso lo Studium fiorentino. Il ravennate Giovanni Malpaghini fu copista di Petrarca in gioventù, seguì il papa ad Avignone nel 1370, provò - invano - ad essere assunto da Carlo Malatesta di Rimini nel 1393, e fu docente di retorica presso lo Studio Fiorentino fra 1394 e $1400^{24}$. Poggio Bracciolini (13801459) è al contempo un cacciatore di manoscritti classici e un raffinato interprete di Lucrezio. Entra in contraddittorio con Lorenzo Valla riguardo le distinte autorità degli antichi e delle Sacre Scritture, attende diligentemente alle sue mansioni presso la curia pontificia per cinquant'anni (ascendendo fino al grado di segretario apostolico), e intrattiene un fitto scambio epistolare con i suoi sodali fiorentini ${ }^{25}$. Serve sotto ben sette papi, ma si considera sempre cittadino fiorentino e allievo di Salutati. Sostiene risolutamente gli studia bumanitatis, ridicolizza i costumi di prelati e principi, ma coltiva l'amicizia di Cosimo de' Medici, Lionello d'Este e di molti altri potenti del suo tempo.

Esistono anche teorici politici che non si schierano per nessuna delle due parti ${ }^{26}$. Fra costoro troviamo Riccobaldo Riccobaldi di Ferrara (1246-1320), lo storico Padovano del XIV secolo Guglielmo Cortusi e Tolomeo Fiadoni da Lucca (c. 12361327). Quest'ultimo studia con Tommaso d'Aquino ed è noto per il suo De regimine principum. Difende il potere ierocratico del papa contro il Sacro Romano Impero e allo stesso tempo propone il regime repubblicano come l'unica alternativa al dispotismo. Marsilio da Padova nel suo Defensor pacis (1324) sottolinea a sua volta l'importanza delle leggi sopra qualsiasi forma di governo per garantire pace e giustizia ai cittadini e afferma che qualsiasi autorità politica è legittima nella misura in cui riesce a sanare i conflitti. Sebbene sia considerato uno fra i primi a enunciare i principi democratici, la sua nozione presuppone che «il governo supremo in una città o stato deve risiedere in un'unica persona» ${ }^{27}$. Questi sono i nomi più illustri di un movimento, che come quello Milanese o come i letterati che orbitano intorno ai pontefici romani, e ai signori padani, fanno dell'umanesimo non solo un fenomeno politicamente trasversale, ma anche un'espressione tipica della cultura cortigiana.

Molti umanisti - di ogni orientamento politico - condividono lo stesso impegno nel campo dell'educazione e teorizzano metodi pedagogici simili. Il policentrismo umanistico si manifesta anche nella fondazione di scuole a Firenze, Ferrara, Venezia, Mantova, Verona, Milano, Padova, dove si insegna ai giovani delle classi dirigenti e, spesso, agli stessi principi. Da queste scuole usciranno Federico da Montefeltro, Carlo Gonzaga e altri «principi splendidi, donne eccezionali, condottieri intrepidi e sapienti,

${ }^{24}$ Cfr. Maddalena Signorini, "Giovanni Malpaghini”, in Dizionario Biografico degli italiani, LXVIII (Roma: Istituto della Enciclopedia Italiana, 2007), ad vocem. Disponibile in www.treccani.it [data di consultazione: $28 / 2 / 2021]$.

${ }^{25}$ Vedi Stephen Greenblatt, The Swerve. How the World Became Modern (New York-Londra: W. W. Norton, 2011), 19.

${ }^{26}$ Jones, The Italian, 647.

${ }_{27}$ Marsilio da Padova, The Defensor Pacis, ed. Alan Gewirth (New York: Columbia University Press, 1956), 427. 
capi della Chiesa, predicatori e santi, dotti insigni, maestri» e tanti altri ${ }^{28}$. Il rinnovamento pedagogico prende prevalentemente la forma di rinnovamento dei curricula e di istituti culturali, come le accademie (quasi sempre adunanze di dotti presso una corte). Per l'inizio del Quattrocento hanno sviluppato un coerente programma educativo fondato sullo studio degli autori greci e latini. Raramente gli umanisti impartiscono il proprio insegnamento alle università, ma creano piuttosto scuole private per i signori e per le élites urbane. Se l'università rappresenta l'istituzione educativa tipica della città medievale, le scuole umanistiche gravitano invece intorno alle corti. «The ideal of humanist education was not the product of the free City, or of the University, but of the Court. [...] Only at a court like Mantova or Ferrara could humanism exhibit its complete educational ideals» ${ }^{29}$.

È questo il caso di Giovanni Conversini a Padova per i da Carrara dopo il 1338, e anche Gasparino Barzizza apre una scuola a Padova nel 1408 dopo qualche anno di controversa docenza all'università di Pavia. Nel 1422 Barzizza lascia Padova, per recarsi alla corte viscontea di Milano. Nel 1423 Vittorino da Feltre accetta l'invito a Mantova del marchese Gian Francesco Gonzaga come istitutore dei propri figli ${ }^{30}$. Prima di questo approdo era stato protonotario e anche lui docente universitario a Padova. Ma aveva anche tenuto scuola a Udine e a Venezia. Per 23 anni (fino alla sua morte nel 1446) la sua scuola - la famosa Ca' Zoiosa - ospita studenti da tutta l'Italia (e oltre) e impartisce lo stesso insegnamento a tutti. Uno studio viene fondato a Ferrara dagli Este alla fine del XIV secolo e sarà riformato dal marchese Leonello (a sua volta discepolo di Guarino da Verona) nel 1442. Vittorino a Mantova e Guarino a Ferrara combinano - così si narra - l'avversione verso la corte, alla stretta familiarità col principe entro la corte.

L'educazione «of the Bentivoglio children was under the charge of Francesco del Pozzo of Parma, known as Puteolano, a humanist of distinction whose presence in Bologna was a stimulus to the intellectual life of the city». Annibale e Alessandro Bentivoglio sono discepoli di Filippo Beroaldo e un terzo umanista - Giovanni Garzoni - racconta che la sua De eruditione principum è frutto delle sue discussioni con Giovanni Bentivoglio $^{31}$. Nelle scuole di corte si insegna il latino e il greco, l'eloquenza e l'etica, ma anche a danzare, andare a cavallo e a tirare di scherma per formare il vir bonus. Il gentiluomo è, ancor prima di quelle gesuitiche, il prodotto delle scuole umanistiche ${ }^{32}$ : egli deve eccellere sia nelle arti fisiche, che contemplative e - soprattutto - deve seguire le norme castiglionesche della grazia. I ceti dominanti del XV secolo assorbono gli studia bumanitatis principalmente da queste scuole private. Dal Portogallo alla Polonia, principi e aristocratici aspirano a ricevere una simile educazione. Oltre alle signorie dell'Italia settentrionale, l'umanesimo è infatti un fenomeno connotativo delle monarchie.

\footnotetext{
${ }^{28}$ Cfr. Eugenio Garin, L'educarione in Europa, 1400/1600 [1957] (Roma-Bari: Laterza, 1976), 130.

${ }^{29}$ William H. Woodward, Vittorino da Feltre and Other Humanist Educators (Cambridge: Cambridge University Press, 1890), 244.

30 Vedi ibidem, 1-91.

${ }^{31}$ Cecilia Ady, The Bentivoglio of Bologna. A Study in Despotism (Oxford: Oxford University Press, 1937), 144.

32 Cfr. Woodward, Vittorino, XII-XVI.
} 
Il riuso dei classici si prefigge di affinare le virtù del principe. Pontano, ma anche Vergerio, Vittorino da Feltre e gli altri istitutori coltivano il sogno di formare un principe umanista, e - perciò - buon reggitore poiché infuso della sapienza e dell'etica classica.

Le lettere aggiungono così alla funzione civilizzatrice l'utilità politica, in quanto elemento primario della capacità di ben governare. Le scuole degli umanisti hanno come allievi sia il principe, che essi introducono alla comunione fra lettere, morale e buoni costumi, che il guerriero feudale, del quale mirano a incivilire i modi. Alle armi, ornamento del sovrano e del cavaliere medievale, gli umanisti sostituiscono le lettere, l'estetica e le virtù degli antichi. In tal senso la preparazione alla vita civile e l'institutio del principe e del nobile segnano due tappe di uno stesso percorso e non esiste soluzione di continuità fra la trattatistica che si rivolge ai patriziati cittadini e i testi cortigiani. I trattati di pedagogia, le scuole e le accademie umanistiche, $i$ loro piani di studio, e i loro docenti contribuiscono al radicamento del classicismo allinterno della civiltà di corte preparando così il terreno per il suo trionfo come universale cultura della politica e della distinzione sociale.

Il De ingenuis moribus et liberalibus adolescentiae studiis (1402) di Pier Paolo Vergerio è quasi un manifesto di questi nuovi orientamenti dell'insegnamento umanistico. Nonostante il suo ideale sia ancora la vita civile, assimilata a Firenze, il suo fine consiste nell'educare i principi, assieme ai loro segretari e ministri, insieme ai patrizi - banchieri e mercanti. Scrivendo a Firenze venti anni dopo la Laudatio, e rivolgendo la propria attenzione all'educazione delle donne, anche Leonardo Bruni perora metodi analoghi ed auspica risultati simili per l'insegnamento umanistico nel suo De studiis et litteris $(1422)^{33}$. La maggior parte dei testi degli umanisti sono del resto dedicati a principi o a membri di famiglie nobili. Il De remediis utriusque fortunae (1366) di Petrarca è dedicato ad Azzo da Correggio signore di Parma e Bruni dedica il De studiis alla moglie di Galeazzo Malatesta. Pier Paolo Vergerio dedica il De ingenuis moribus a Ubertino da Carrara signore di Padova. E sempre Vergerio, al servizio dei da Carrara, scrive le Vitae principum carrarensium. E potremmo continuare.

Tutto questo scalfisce appena la superficie di un fenomeno ampio e profondo. Ugualmente utili a tutti gli uomini di potere sono gli studi storici; essi offrono indistintamente ai cittadini e ai signori stimuli e ammonimenti per ben governare. Un campo sterminato da indagare sarebbe quello della rinascita degli antichi nei cicli figurativi. Qui vi facciamo solo un richiamo per il ruolo svolto dagli umanisti nel compilare repertori mitografici, nel riproporre storie, eroi e fabulae classiche in forma tale da essere facilmente utilizzabili dagli artisti e da fungere da vademecum per la comprensione dei fruitori ${ }^{34}$.

Un'ampia letteratura umanistica sulle immagini sostiene questo linguaggio. Attraverso cataloghi enciclopedici, vite di eroi e libri di emblemi, la mitologia classica si sposta dall'Italia all'Europa delle monarchie e delle aristocrazie. Il boom della mitografia per l'uso di artisti e mecenati contribuisce a soddisfare la domanda. Come al solito, la Genealogia deorum gentilium (1370) di Boccaccio fu un buon punto di partenza. Coluccio Salutati aveva scritto specificatamente su Ercole nel suo De laboribus Herculis,

\footnotetext{
${ }^{33}$ Cfr. Garin, L'educazione.

${ }^{34}$ Cfr. Jan Seznec, The Survival of Pagan Gods [1940] (New York: Pantheon Books, 1953).
} 
compilato tra il 1375 e il 1406 e primo di una lunga serie di opere a contenuto mitografico. A questo genere ha contribuito anche Lilio Gregorio Giraldi da Ferrara con una vita di Ercole (1539) e un trattato sulle storie degli dei antichi (1548). Ma il testo canonico era di Vincenzo Cartari, protetto del Duca di Ferrara, con le sue Le imagini con la sposiz̨ione dei dèi antichi (1556). Qualche anno dopo fu la volta del poeta e umanista Natale Conti e delle sue Mythologiae (1567) in dieci libri. Le nuove caratteristiche degli antichi dei sono state codificate e moltiplicate da queste "Bibbie profane", manuali in cui sono state catturate le loro storie e immagini. La citata trilogia fu in particolare pubblicata in rapida successione tra il 1548 e il $1556^{35}$.

Anche qui non ci sono confini netti: gli antichi (quelli utili al caso) compaiono sia nelle sale dei palazzi pubblici che in quelle delle residenze signorili. Gli dei sono sempre gli stessi, ma cambiano solo le loro selezioni per accordarsi con i diversi contesti. Un altro settore nel quale si riverbera il ruolo degli umanisti è quello dei miti sull'origine delle civitas, molti dei quali affondano le proprie radici nell'antichità romana. Virgilio è quasi equiparato a un santo patrono nella sua natale Mantova: Antenore (consigliere di re Priamo a Troia) viene dichiarato fondatore di Padova dopo il supposto ritrovamento della sua tomba; una statua di una Venere nuda viene installata sulla facciata del Palazzo Pubblico di Siena; ed Ercole o Augusto compaiono frequentemente come numi tutelari o antenati di molte città.

Anche in questi casi il repubblicanesimo non è l'unico colore dell'umanesimo. L'umanesimo è un movimento con molte voci dialettiche. La riverenza verso il simbolismo dell'antichità non ha una univoca connotazione politica ${ }^{36}$. Dal XIV secolo corti e comuni formano una sorta di république des lettres et des images. E questo tanto a livello europeo che nazionale. Quando, nel 1477, con la battaglia di Nancy, svanisce il progetto di un regno di Borgogna, in Europa circolavano già ampiamente i modelli umanistici italiani. La cultura elaborata e proveniente dalle corti italiane già coesiste $\mathrm{e}$ contende il ruolo egemonico ai modelli borgognoni ${ }^{37}$. Anzi, il processo era iniziato da tempo. Sulla diffusione europea dell'umanesimo italiano (prevalentemente quello cortigiano) esiste un'estesa letteratura. Dalla fine del XIV secolo Petrarca inizia ad esercitare un'enorme influenza sulla letteratura europea e il petrarchismo diventa una delle principali esportazioni letterarie dell'Italia ben oltre i confine dell'umanesimo ${ }^{38}$. La cattività avignonese (1307-1378) aveva già portato lo stesso Petrarca e molti artisti italiani in Francia. Il Concilio di Costanza (1414-1418), convocato per ricomporre lo Scisma d'Occidente, aveva a sua volta visto il concorso di umanisti da ogni parte d'Europa.

\footnotetext{
${ }^{35}$ De deis gentium varia et multiplex historia in qua simul de eorum imaginibus et cognominibus agitur, di Lilio Gregorio Giraldi (Basilea: Oporinus, 1548); Mythologiae sive explicationis fabularum libri decem, di Natale Conti (Venezia: Aldus, 1551); e Le imagini colla sposizione degli dei degli antichi, di Vincenzo Cartari (Venezia: Marcolini, 1556).

36 Jones, The Italian, 649.

${ }^{37}$ Vedi Johan Huizinga, Le déclin du Moyen Âge (Parigi: Payot, 1919) e Marina Belozerskaya, Retbinking the Renaissance. Burgundian Arts across Europe (Cambridge: Cambridge University Press, 2002).

38 Roberto Weiss, The Spread of Italian Humanism (Amherst: Da Valley Books, 1964), 1.
} 
In generale, gli umanisti italiano stabiliscono un nuovo curriculum e redigono testi che saranno ristampati molte volte in tutta Europa: ovunque contribuiscono al revival dell'antichità e non pochi di loro viaggiano ampiamente all'estero. I due principali veicoli per la diffusione dell'umanesimo sono le persone e i libri. Ovunque essi si rechino, stabiliscono nuovi movimenti culturali e attraggono studenti e studiosi in Italia. Poggio Bracciolini serve per tre anni (1418-1422) come segretario di Henry Beaufort, vescovo di Winchester. Gli umanisti sono una nuova specie di studioso che viaggia costantemente al seguito di protettori e indizi bibliografici, ed è interconnessa da molteplici fili di amicizia, interessi accademici, e scambio culturale. «Molti dotti italiani entrarono al servizio di principi o dignitari» di tutta Europa «come istitutori o segretari, bibliotecari, poeti o storici di corte» ${ }^{39}$. Nel 1383 la regina Elisabetta di Ungheria offre a Giovanni Conversino il posto di notarius mayor nella Repubblica di Ragusa, all'epoca stato vassallo ungherese. L'umanista toscano Filippo Buonaccorsi, detto Callimaco (1437-1496), soggiorna alla corte di Cracovia per buona parte della sua vita. Nel 1470, la Polonia è l'approdo della sua fuga dopo aver preso parte nel tentato assassinio di papa Paolo II nel 1468. La sua nuova vita da esule lo porta a divenire tutore dei figli di Casimiro IV Jaghellone, a svolgere delicati incarichi diplomatici e ad essere assunto come segretario reale nel 1474. Nel 1489 era stato anche tra i cofondatori della Sodalitas Litteraria Vistulana che raccoglie in forma di accademia una eletta cercia di ammiratori del Rinascimento italiano e dell'antichità. Dopo il Concilio di Costanza, che egli contribuisce a organizzare, Pier Paolo Vergerio, il quale aveva appreso i valori dell'umanesimo civile da Salutati e Bruni a Firenze, segue l'imperatore Sigismondo, e continua il suo viaggio finendo i propri giorni nel 1444 a Buda alla corte dei re d'Ungheria ${ }^{40}$.

Restiamo negli stessi anni, ma spostiamoci in Inghilterra, dove il regolare scambio diplomatico con la curia pontificia «prompted the entry of humanists into government service». L'influso era già iniziato in precedenza, ma è con Enrico VII (1485-1509) «that the humanists really came to court for the first time». Fra gli altri, il re impiega Pietro Carmeliano da Brescia «as his Latin secretary» e sempre Enrico inizia «to give his children a humanist education» ${ }^{41}$. Filippo Beroaldo (1453-1505), professore di poesia e retorica all'università di Bologna dal 1472 (sotto i Bentivoglio), viaggia a Parigi nel 1475 e qui stringe amicizia col filosofo Robert Gaguin, sul cui pensiero eserciterà una forte influenza. Publio Fausto Andrelini, di nove anni più giovane di Beroaldo, fu anch'egli poeta e intimo amico di Erasmo. Per recarsi in Francia lascia la corte del cardinale Ludovico Gonzaga nel 1488. Contribuisce alla diffusione dell'umanesimo dalla sua cattedra all'università di Parigi, ottenuta nel 1489, e - in seguito - dalla sua carica di poeta di corte sotto Carlo VIII. Infine, Gian Giorgio Trissino (1478-1550), dopo aver studiato greco with Demetrio Calcondila, diviene un

\footnotetext{
39 Paul Oskar Kristeller, "La diffusione europea dell'umanesimo italiano" [1962], in Il pensiero e le arti nel Rinascimento, ed. Paul Oskar Kristeller (Roma: Donzelli, 1998), 75-94.

${ }^{40}$ John Monfasani, "Umanesimo italiano e cultura europea", in Il Rinascimento italiano e l'Europa. I. Storia e Storiografia, ed. Marcello Fantoni (Costabissara: Angelo Colla, 2006), 49-70.

${ }^{41}$ Roy Strong, The Spirit of Britain. A Narrative History of the Arts (Londra: Jonathan Cape Ltd., 1999),
} 133. 
favorito di Papa Leone X, viaggia in Germania come ambasciatore, e nel 1532 viene nominato conte dall'imperatore Carlo V. Questa è sola la punta dell'iceberg.

L'umanesimo cortigiano costituisce il fondamento di quel processo che, portando alla formulazione di una nuova cultura in Italia, conferirà a questa le caratteristiche che ne determineranno l'irradiamento europeo sulla base di una forza catalizzatrice che è eminentemente politica. Le 'piccole' corti italiane del Quattrocento funzionano, in questo, da incubatrici dei nuovi modelli di civilizzazione per le grandi corti europee dell'Antico regime, e - alla lunga - tutto ciò andrà a confluire negli elementi fondanti l'identità europea.

A questo punto, mi piace aprire un'ultima breve finestra esemplificativa sull'arrivo dell'umanesimo e dell'arte italiana in Ungheria. Ovunque arrivassero gli umanisti, introducevano i germi di una nuova filosofia e sensibilità. Prima che l'Europa scoprisse l'Italia nell'epoca delle guerre per la sua conquista, gli umanisti avevano letteralmente aperto la strada a una rivoluzione nei modelli culturali e nel linguaggio della politica in Ungheria. Il monarca più impegnato nell'adozione dei nuovi paradigmi fu Mattia Corvino, re d'Ungheria dal 1458 al 1490, re di Boemia dal 1469 e duca d'Austria dal 1486. Sotto la corona di Mattia l'umanesimo fu trapiantato alla corte ungherese e artisti, architetti e scultori italiani accorsero a Buda, a Visegrád e a Esztergom per ricostruire e decorare i palazzi reali. I legami con l'umanesimo italiano erano già esistenti attraverso l'inclusione nel regno angioino nel XIV secolo (1308-1382) e un ruolo deve essere attribuito anche alla sposa italiana di Mattia, Beatrice d'Aragona.

Anche il suo predecessore Sigismondo di Lussemburgo (1404-1437) aveva già avviato il processo dopo la sua visita a Roma, dove conobbe Ciriaco d'Ancona e Poggio Bracciolini. Sulla scia di questo viaggio, molti umanisti si recarono alla corte di Buda: tra gli altri Ambrogio Traversari, Antonio Loschi e Francesco Filelfo. Sebbene non avesse mai visitato l'Ungheria, per oltre 20 anni Marsilio Ficino stabilì uno stretto rapporto con la corte ungherese con il suo scambio epistolare con Janus Pannonius, un membro di spicco del seguito di Corvino. Ficino introdusse il Neoplatonismo e inviò manoscritti filosofici consegnati dall'amico Francesco Bandini, tra cui il Trattato dell'architettura del Filarete nel 1487 o $1488^{42}$. Il re si circondò di umanisti. Péter Váradi, che aveva studiato a Bologna nel 1460, fu nominato segretario e cancelliere nel 1475, Janus Pannonius, che aveva trascorso la maggior parte della sua vita alle corti italiane e che aveva studiato a Padova (1447-1458), fu uno dei collaboratori più stretti, e Jacobus Pisone dalla Transilvania, poeta e diplomatico, fu un importante intellettuale di corte dopo aver trascorso quasi un decennio a Roma. Anche il fiorentino Francesco Bandini finì per stabilirsi a Buda al seguito di Beatrice d'Aragona nel 1476. Con il tempo Bandini divenne «il principale consigliere del re su questioni artistiche (soprattutto architettoniche)» ${ }^{43}$. Infine, Antonio Bonfini, autore di la Rerum Ungaricarum

42 Vedi Valery Rees, "Marsilio Ficino and the Rise of Philosophic Interests in Buda", in Italy \& Hungary. Humanism and Art in the Early Renaissance, ed. Péter Farbaky e Louis A. Waldman (Firenze: Olschki, 2011), 127-148.

43 Péter Farbaky, "Florence and/or Rome? The Origins of Early Renaissance Architecture in Hungary", in Italy \& Hungary. Humanism and Art in the Early Renaissance, ed. Péter Farbaky e Louis A. Waldman (Firenze: Olschki, 2011), 345-367, 347. 
decades, fu nominato storico reale nel 1488. Lo stesso Mattia fu educato dall'umanista croato János Vitéz (che aveva studiato in Italia) e intrattenne una corrispondenza con filosofi italiani. La Biblioteca Corviniana getta ulteriore luce sulle inclinazioni culturali di Mattia, essa conteneva molta letteratura umanistica, che - secondo i testimoni - avrebbe letto spesso.

Tutti gli umanisti in Ungheria «hanno avuto un impatto duraturo sul trattamento delle arti visive» e dell'architettura ${ }^{44}$. Gli umanisti ispirarono un mecenatismo etico e furono educatori nelle arti. Alcuni di loro svolsero un ruolo chiave nell'età dell'oro del regno di Mattia: in particolare, Antonio Bonfini dopo il 1486 dalla sua posizione di lettore ad alta voce alla regina; Francesco Arrigoni, per otto anni alla corte di Buda; e Francesco Bandini de Baroncelli. Quest'ultimo divenne ambasciatore e svolse un ruolo importante come catalizzatore del Neoplatonismo e nell'introduzione dell'arte rinascimentale. Durante il suo precedente soggiorno a Napoli (1474-1476) conobbe la cerchia di Pontano (anche Beatrice apparteneva alla sua cerchia) e assorbì il concetto di magnificentia a cui in seguito convertì il re. Bonfini elabora questa idea in una apologia del re Mattia dove afferma che la sua «magnificentia ha superato gli edifici e le opere» degli antichi imperatori romani a causa delle sue enormi spese e perché ha invitato artisti italiani a stabilire «un'architettura autenticamente antica» ${ }^{45}$.

44 Thomas DaCosta Kaufmann, Court, Cloister \& City. The Art and Culture of Central Europe, 1450-1800 (Chicago: University of Chicago Press, 1995), p.65.

45 Rózsa Feuer-Tóth, Art and Humanism in Hungary in the Age of Matthias Corvinus (Budapest: Akadémiai Kiadó, 1990), 69. 


\section{BIBLIOGRAFIA}

Ady, Cecilia, The Bentivoglio of Bologna. A Study in Despotism (Oxford: Oxford University Press, 1937).

Baldassarri, Stefano, "Introduction", in Giannozzo Manetti, Biograpbical Writings, ed. Stefano Baldassarri e Rolf Bagemihl (Cambridge-Londra: Harvard University Press, 2003), VII-XIX.

Baron, Hans, The Crisis of the Early Italian Renaissance. Civic Humanism and Republican Liberty in an Age of Classicism and Tyranny (Princeton: Princeton University Press, 1955).

Becker, Marvin, Florence in Transition, 2 voll. (Baltimore: Johns Hopkins University Press, 1967-1968), https://doi.org/10.1353/book.67860, https://doi.org/10. 1353/book.67859.

_. "The Florentine Territorial State and Civic Humanism in the Early Renaissance", in Florentine Studies. Politics and Society in Renaissance Florence, ed. Nicolai Rubinstein (Evanston: Northwestern University Press, 1968), 109-139.

Belozerskaya, Marina, Rethinking the Renaissance. Burgundian Arts across Europe (Cambridge: Cambridge University Press, 2002).

Bentley, Jerry, Politics and Culture in Renaissance Naples (Princeton: Princeton University Press, 2014), https://doi.org/10.1515/9781400858811.

Boschetto, Luca, "L'esilio volontario di Manetti", in Dignitas et excellentia hominis. Atti del Convegno Internazionale di Studi su Giannozzo Manetti, ed. Stefano Baldassarri (Firenze: Le Lettere, 2008), 117-146.

Brown, Allison, "De-masking Renaissance Republicanism", in Renaissance Civic Humanism. Reappraisals and Reflections, ed. James Hankins (Cambridge: Cambridge University Press, 2000), 179-199, https://doi.org/10.1017/CBO9 $\underline{780511558474.007 .}$.

Brucker, Gene, The Civic World of Early Renaissance Florence (Princeton: Princeton University Press, 1977), https://doi.org/10.1515/9781400847853.

Burdach, Konrad, Riforma, Rinascimento, Umanesimo [1935] (Firenze: Sansoni, 1986).

Conversini da Ravenna, Giovanni, Two Court Treatises, ed. Benjamin G. Kohl e James Day (Monaco: Wilhelm Fink Verlag, 1987). 
Da Padova, Marsilio, The Defensor Pacis, ed. Alan Gewirth (New York: Columbia University Press, 1956).

Emerton, Ephraim, Humanism and Tyranny. Studies in the Italian Trecento (Cambridge: Harvard University Press, 1925), https://doi.org/10.4159/harvard.97806743 $\underline{33178}$.

Farbaky, Péter, "Florence and/or Rome? The Origins of Early Renaissance Architecture in Hungary", in Italy \& Hungary. Humanism and Art in the Early Renaissance, ed. Péter Farbaky e Louis A. Waldman (Firenze: Olschki, 2011), 345-367.

Feuer-Tóth, Rózsa, Art and Humanism in Hungary in the Age of Matthias Corvinus (Budapest: Akadémiai Kiadó, 1990).

Garin, Eugenio, "La letteratura degli umanisti", in Storia della Letteratura Italiana. III. Il Quattrocento e l'Ariosto (Milano: Garzanti, 1966), 7-353.

_, L'educazione in Europa, 1400/1600 [1957] (Roma-Bari: Laterza, 1976).

Greenblatt, Stephen, The Swerve. How the World Became Modern (New York-Londra: W. W. Norton, 2011).

Huizinga, Johan, Le déclin du Moyen Âge (Parigi: Payot, 1919).

Jones, Philip, The Italian City-State. From Commune to Signoria (Oxford: Clarendon Press, 1997).

Kaufmann, Thomas DaCosta, Court, Cloister \& City. The Art and Culture of Central Europe, 1450-1800 (Chicago: University of Chicago Press, 1995).

Kristeller, Paul Oskar, Renaissance Thought II. Papers on Humanism and the Arts (New York: Harper Torchbooks, 1965).

—. "La diffusione europea dell'umanesimo italiano" [1962], in Il pensiero e le arti nel Rinascimento, ed. Paul Oskar Kristeller (Roma: Donzelli, 1998), 75-94.

Martelli, Mario, “Profilo ideologico di Giannozzo Manetti”, Studi Italiani 1 (1989): 5-41.

Martines, Lauro, The Social World of the Florentine Humanists, 1390-1460 (Princeton: Princeton University Press, 1963).

_, Potere e fantasia. Le città stato nel Rinascimento [1979] (Roma-Bari: Laterza, 1981). 
Monfasani, John, "Umanesimo italiano e cultura europea", in Il Rinascimento italiano e l'Europa. I. Storia e Storiografia, ed. Marcello Fantoni (Costabissara: Angelo Colla, 2006), 49-70.

Pocock, J. G. A., The Machiavellian Moment. Florentine Political Thought and the Atlantic Republican Tradition (Princeton: Princeton University Press, 1975).

Pontano, Giovanni, De Magnanimitate [1498], ed. Francesco Tateo (Firenze: Istituto Nazionale di Studi sul Rinascimento, 1969).

Rabil, Albert, Jr., ed., Renaissance Humanism. Foundations, Forms and Legacy. I. Humanism in Italy (Philadelphia: University of Pennsylvania Press, 1988).

Rees, Valery, "Marsilio Ficino and the Rise of Philosophic Interests in Buda", in Italy \& Hungary. Humanism and Art in the Early Renaissance, ed. Péter Farbaky e Louis A. Waldman (Firenze: Olschki, 2011), 127-148.

Ricciardelli, Fabrizio, "Giannozzo Manetti, un intellettuale al potere", in Dignitas et excellentia hominis. Atti del Convegno Internazionale di Studi su Giannozzo Manetti, ed. Stefano Baldassarri (Firenze: Le Lettere, 2008), 279-300.

Seigel, Jerrold, “'Civic Humanism' or Ciceronian Rhetoric? The Culture of Petrarch and Bruni", Past and Present 34 (1966): 3-48, https://doi.org/10.1093/past/34 .1 .3 .

Seznec, Jan, The Survival of Pagan Gods [1940] (New York: Pantheon Books, 1953).

Signorini, Maddalena, "Giovanni Malpaghini”, in Diz̧ionario Biografico degli Italiani, LXVIII (Roma: Istituto della Enciclopedia Italiana, 2007), ad vocem. Disponibile in www.treccani.it [data di consultazione: 28/2/2021].

Skinner, Quentin, The Foundations of Modern Political Thought. I. The Renaissance (Cambridge: Cambridge University Press, 1978), https://doi.org/10.1017/C BO9780511817878.

Strong, Roy, The Spirit of Britain. A Narrative History of the Arts (Londra: Jonathan Cape Ltd., 1999).

Trinkaus, Carl, In Our Image and Likeness. Humanity and Divinity in Italian Humanist Thought, 2 voll. (Chicago: University of Chicago Press, 1970).

Weiss, Roberto, The Spread of Italian Humanism (Amherst: Da Valley Books, 1964). 
Woodward, William H., Vittorino da Feltre and Other Humanist Educators (Cambridge: Cambridge University Press, 1890).

Recibido: 28 de febrero de 2021

Aprobado: 9 de abril de 2021 